\title{
A Review of Wireless Body Area Networks for Medical Applications
}

\author{
Sana ULLAH ${ }^{1}$, Pervez KHAN ${ }^{1}$, Niamat ULLAH ${ }^{1}$, Shahnaz SALEEM ${ }^{2}$, \\ Henry HIGGINS ${ }^{3}$, Kyung Sup KWAK ${ }^{1}$ \\ ${ }^{I}$ Graduate School of Telecommunication Engineering, Inha University Incheon, Nam-Gu, South Korea \\ ${ }^{2}$ Graduate School of Computer Engineering, Inha University Incheon, Nam-Gu, South Korea \\ ${ }^{3}$ Zarlink Semiconductor Company, Portskewett, Caldicot, United Kingdom \\ Email: \{sanajcs, pervaizkanju,roshnee13\}@hotmail.com,niamatnaz@gmail.com,kskwak@inha.ac.kr, \\ henry.higgins@zarlink.com \\ Received March 8, 2009; revised May 16, 2009; accepted July 27, 2009
}

\begin{abstract}
Recent advances in Micro-Electro-Mechanical Systems (MEMS) technology, integrated circuits, and wireless communication have allowed the realization of Wireless Body Area Networks (WBANs). WBANs promise unobtrusive ambulatory health monitoring for a long period of time, and provide real-time updates of the patient's status to the physician. They are widely used for ubiquitous healthcare, entertainment, and military applications. This paper reviews the key aspects of WBANs for numerous applications. We present a WBAN infrastructure that provides solutions to on-demand, emergency, and normal traffic. We further discuss in-body antenna design and low-power MAC protocol for a WBAN. In addition, we briefly outline some of the WBAN applications with examples. Our discussion realizes a need for new power-efficient solutions towards in-body and on-body sensor networks.
\end{abstract}

Keywords: Wireless Body Area Networks, Low Power MAC, Body Sensor Networks, BSN, WBAN

\section{Introduction}

Cardiovascular disease is the foremost cause of death in the United States (US) and Europe since 1900. More than ten million people are affected in Europe, one million in the US, and twenty two million people in the world [1-3]. The number is projected to be triple by 2020. The ratio is $17 \%$ in South Korea and $39 \%$ in UK [4-5]. The healthcare expenditure in the US is expected to increase from \$2.9 trillion in 2009 to $\$ 4$ trillion in 2015 [6]. The impending health crisis attracts researchers, industrialists, and economists towards optimal and quick health solutions. The non-intrusive and ambulatory health monitoring of patient's vital signs with real time updates of medical records via internet provides economical solutions to the health care systems.

A WBAN contains a number of portable, miniaturised, and autonomous sensor nodes that monitors the body function for sporting, health, entertainment, and emergency applications. It provides long term health moni-

\footnotetext{
${ }^{1}$ Ventricular tachyarrhythmia are abnormal patterns of electrical activitv originating within ventricular tissue.
}

toring of patients under natural physiological states without constraining their normal activities. In-body sensor networks allow communication between implanted devices and remote monitoring equipments. They are used to collect information from Implantable Cardioverter Defibrillators (ICDs) in order to detect and treat ventricular tachyarrhythmia ${ }^{1}$ and to prevent Sudden Cardiac Death (SCD) [7].

A number of ongoing projects such as CodeBlue, MobiHealth, and iSIM have contributed to establish a proactive WBAN system [8-10]. A system architecture presented in [11] performs real-time analysis of sensor's data, provides real-time feedback to the user, and forwards the user's information to a telemedicine server. UbiMon aims to develop a smart and affordable health care system [12]. MIT Media Lab is developing MIThril that gives a complete insight of human-machine interface [13] HIT lab focuses on quality interfaces and innovative wearable computers [14]. NASA is developing a wearable physiological monitoring system for astronauts called LifeGuard system [15]. IEEE 802.15.6 aims to provide low-power in-body and 


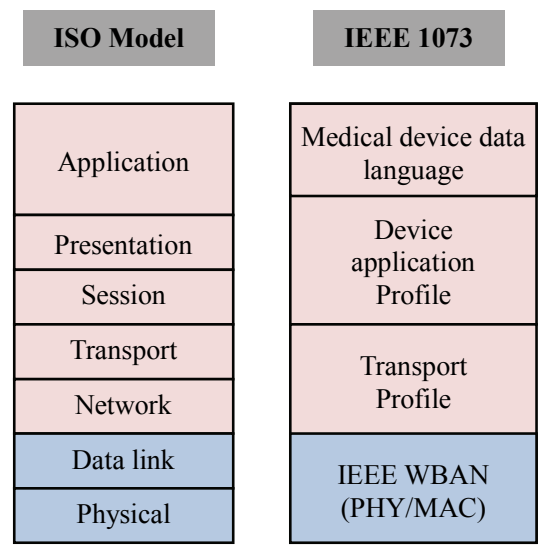

Figure 1. Model ISO and IEEE 1073.

on-body wireless communication standards for medical and non-medical applications [16]. IEEE 1073 is working towards a seven layers solution for wireless communication in a WBAN [17]. Figure 1 shows IEEE 1073 model.

The rest of the paper is organized into five sections. Section 2 presents a WBAN infrastructure for medical and non-medical applications. Section 3 and 4 discuss in-body antenna design and low-power MAC protocol for a WBAN. Section 5 outlines some of the WBAN applications. The final section concludes our work.

\section{WBAN Infrastructure}

A WBAN consists of in-body and on-body nodes that continuously monitor patient's vital information for diagnosis and prescription. Some on-body nodes are used for multimedia and gaming applications.

A WBAN uses Wireless Medical Telemetry Services (WMTS), unlicensed Industrial, Scientific, and Medical (ISM), Ultra-wideband (UWB), and Medical Implant Communications Service (MICS) bands for data transmission. WMTS is a licensed band used for medical telemetry system. Federal Communication Commission (FCC) urges the use of WMTS for medical applications due to fewer interfering sources. However, only authorized users such as physicians and trained technicians are eligible to use this band. Furthermore, the restricted WMTS (14 MHz) bandwidth cannot support video and voice transmissions. The alternative spectrum for medical applications is to use $2.4 \mathrm{GHz}$ ISM band that includes guard bands to protect adjacent channel interference. A licensed MICS band (402-405 MHz) is dedicated to the implant communication.

Figure 2 shows the proposed WBAN infrastructure for medical and non-medical applications.

The WBAN traffic is categorized into On-demand, Emergency, and Normal traffic. On-demand traffic is initiated by the coordinator or doctor to acquire certain

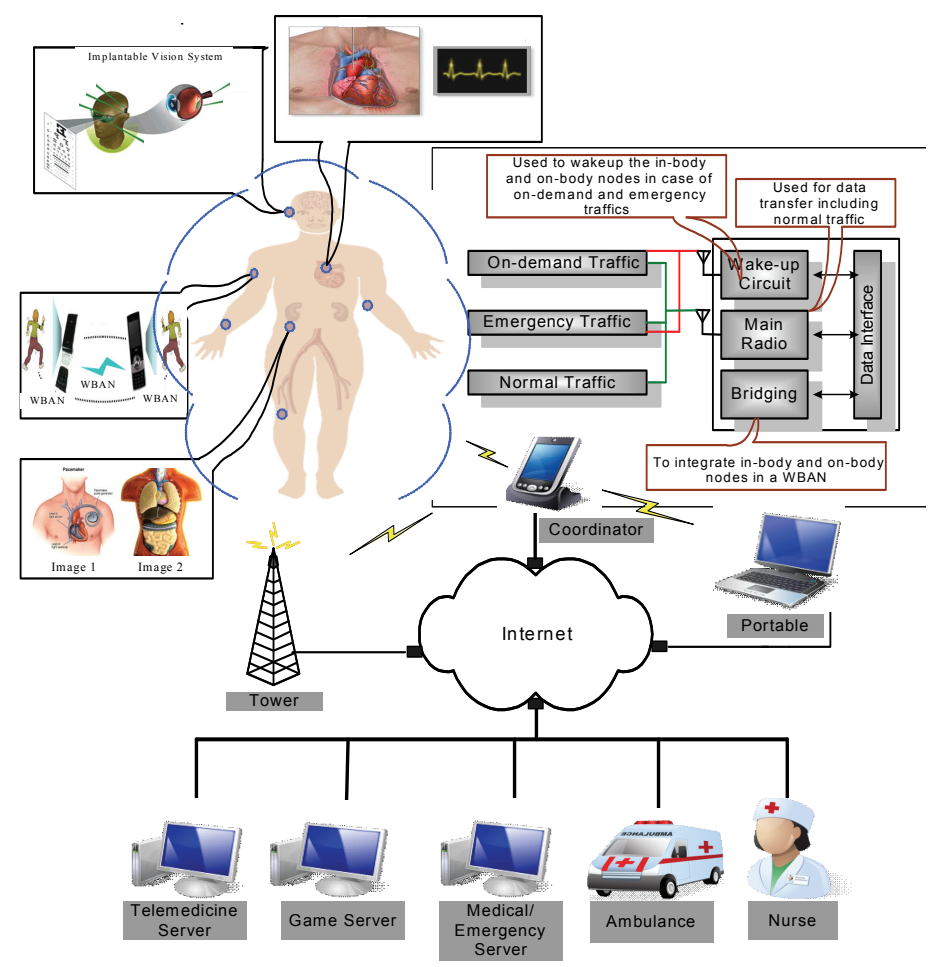

Figure 2. A WBAN infrastructure for medical and non-medical applications. 
information, mostly for the purpose of diagnostic recommendations. This is further divided into continuous (in case of surgical events) and discontinuous (when occasional information is required). Emergency traffic is initiated by the nodes when they exceed a predefined threshold and should be accommodated in less than one second. This kind of traffic is not generated on regular intervals and is totally unpredictable. Normal traffic is the data traffic in a normal condition with no time critical and on-demand events. This includes unobtrusive and routine health monitoring of a patient and treatment of many diseases such as gastrointestinal tract, neurological disorders, cancer detection, handicap rehabilitation, and the most threatening heart disease. The normal data is collected and processed by the coordinator. The coordinator contains a wakeup circuit, a main radio, and a bridging function, all of them connected to a data interface. The wakeup circuit is used to accommodate ondemand and emergency traffic. The Bridging function is used to establish a logical connection between different nodes working on different frequency bands. The coordinator is further connected to telemedicine, game, and medical servers for relevant recommendations.

\section{In-Body Antenna Design}

The band designated for in-body communication is MICS and is around 403MHz. The wavelength of this frequency in space is $744 \mathrm{~mm}$ so a half wave dipole will be $372 \mathrm{~mm}$. Clearly, it is not possible to include an antenna of such dimensions in a body [19]. These constraints make the available size much smaller than the optimum.

The electrical properties of a body affect the propagation in several ways. First, the high dielectric constant increases the "electrical length" of E-field antennas such as a dipole. Second, body tissue such as muscle is partly conductive and will absorb some of the signal but it can also act as a parasitic radiator. This is significant when the physical antenna is much smaller than the optimum. Typical dielectric constant $\left(\varepsilon_{r}\right)$, conductivity $(\rho)$ and characteristic impedance $Z_{0}(\Omega)$ properties of muscle and fat are shown in Table 1.

1) Dipole Antenna: For a dipole of length $10 \mathrm{~mm}$, at $403 \mathrm{MHz}$, the radiation resistance is $45 \mathrm{~m} . \Omega$ in air. The electrical length of the dipole is increased when surrounded by material of a high dielectric constant such as the body.

2) Loop Antenna: For a loop of $10 \mathrm{~mm}$ diameter the area is $78.5 \mathrm{~mm}^{2}$, this gives the radiation resistance of $626 \mu \Omega$. However, the loop acts, as a "magnetic dipole" producing a more intense magnetic field than a dipole. The loop is of use within the body as the magnetic field is less affected by the body tissue compared to a dipole or a patch and it can be readily integrated into existing structures.

3) Patch Antenna: A patch antenna can be integrated into the surface of an implant. Without requiring much additional volume, the ideal patch will have dimensions as shown in Figure 3 and acts as a $\lambda / 2$ parallel-plate transmission line with an impedance inversely proportional to the width.

The radiation occurs at the edges of the patch, as

Table 1. Body electrical properties [19].

\begin{tabular}{ccccccc}
\hline \multirow{2}{*}{ Frequency } & \multicolumn{4}{c}{ Muscle } & \multicolumn{3}{c}{ Fat } \\
\cline { 2 - 7 } & $\left(\varepsilon_{r}\right)$ & $\rho\left({\left.\mathrm{S} . \mathrm{m}^{-1}\right)}\right)$ & $Z_{0}(\Omega)$ & $\left(\varepsilon_{r}\right)$ & $\rho$ & $Z_{0}(\Omega)$ \\
\hline 100 & 66.2 & 0.73 & 31.6 & 12.7 & 0.07 & 92.4 \\
400 & 58 & 0.82 & 43.7 & 11.6 & 0.08 & 108 \\
900 & 56 & 0.97 & 48.2 & 11.3 & 0.11 & 111 \\
\hline
\end{tabular}

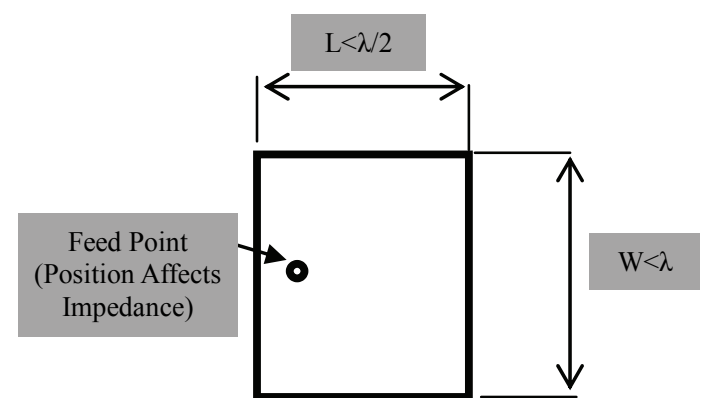

Figure 3. Patch antenna plan view, $\lambda$ in the surrounding medium.

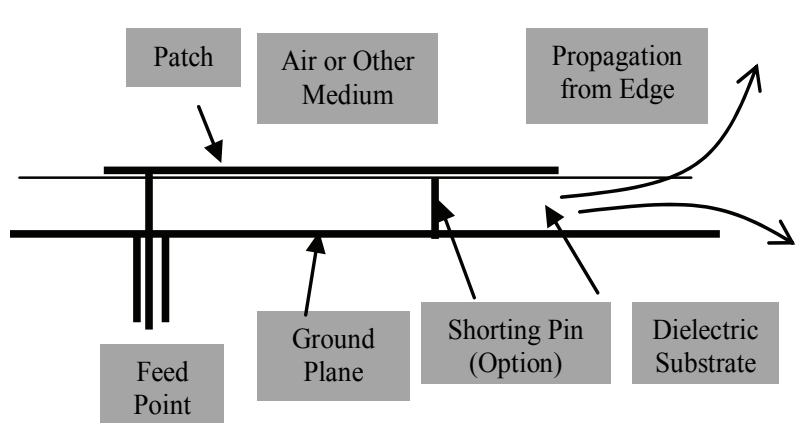

Figure 4. Patch antenna side view. 
shown in Figure 4. For in-body use a full size patch is not an option. However, as it is immersed in a body tissue that has a dielectric constant in the order of 50 , the electrical size of the patch becomes larger than would be in air. An electrically small patch will have low real impedance and therefore impaired performance compared to the ideal one. There are several other options for antenna such as Planar Inverted-F Antenna (PIFA), loaded PIFA, the bow tie, spiral and trailing wire. These antennas may have properties that may make them better suited for some applications.

4) Impedance Measurement: The impedance of the patch and dipole will be affected considerably by being surrounded by the body tissue. The doctor who fits it determines the position of an implant within a body. It may move within the body after fitting. Each body has a different shape with different proportions of fat and muscle that may change with time. This means that a definitive measurement of antenna impedance is of little value. Measuring it immersed in a body phantom can make an approximation of impedance liquid [20]. Using this impedance, the antenna-matching network can be designed with the provision of software controlled trimming as can be done with variable capacitors integrated into the transceiver. The trimming routine should be run on each power up or at regular intervals to maintain optimum performance.

\section{MAC Protocol}

The design and implementation of a low-power MAC protocol for a WBAN is currently a hot research topic. The most challenging task is to accommodate the inbody nodes in a power-efficient manner. Unlike on-body nodes, the in-body nodes are implanted under human skin where the electrical properties of the body affect the signal propagations. The human body is a medium that poses many wireless transmission challenges. The body is composed of several components that are unpredictable and subjected to change.

Li et al. proposed a novel TDMA protocol for an on-body sensor network that exploits the biosignal features to perform TDMA synchronization and improves the energy efficiency [21]. Other protocols like WASP, CICADA, and BSN-MAC are proposed in [22-24]. The performance of a non-beacon IEEE 802.15.4 is investigated in [25], where the authors considered low upload/download rates, mostly per hour. Furthermore, the data transmission is based on periodic intervals that limit the performance to certain applications. There is no reliable support for on-demand and emergency traffic.

The WBAN traffic requires sophisticated low-power techniques to ensure safe and reliable operations. Exist-

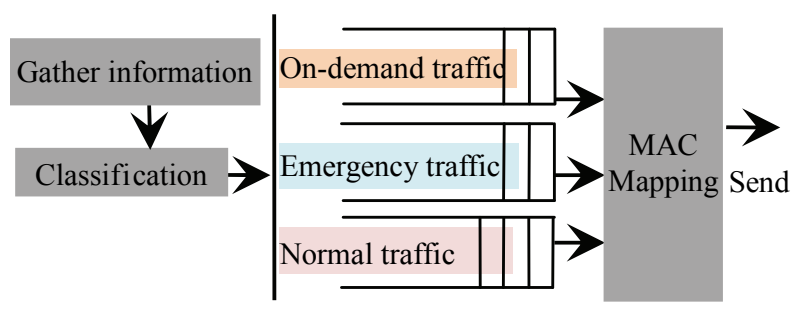

Figure 5. WBAN MAC mapping.

ing MAC protocols such as SMAC [26], TMAC [27], IEEE 802.15.4 [28], and WiseMAC [29] give limited answers to the heterogeneous traffic. The in-body nodes do not urge synchronized wakeup periods due to sporadic medical events. Medical data usually needs high priority and reliability than non-medical data. In case of emergency events, the nodes should access the channel in less than one second [30]. IEEE 802.15.4 Guaranteed Time Slots (GTS) can be utilized to handle time critical events but they expire in case of a low traffic. Furthermore, some in-body nodes have high data transmission frequency than others. Figure 5 shows the required MAC mapping of the WBAN traffic.

The IEEE 802.15.4 can be considered for certain on-body sensor network applications but this does not achieve the required power level of in-body nodes. For critical and non-critical medical traffic, the IEEE 802.15.4 has several power consumption and QoS issues [31-34]. Also, this standard operates in $2.4 \mathrm{GHz}$ band, which allows the possibilities of interference from other devices such as IEEE 802.11 and microwave. Dave et al. studied the energy efficiency and QoS performance of IEEE 802.15.4 and IEEE 802.11e [35] MAC protocols under two generic applications: a wave-form real time stream and a real-time parameter measurement stream [36]. Table 2 shows the Packet Delivery Ratio and the Power (in $\mathrm{mW}$ ) for both applications. The $A C_{-} B E$ and $A C_{-} V O$ represent the access categories voice and best-effort in the IEEE 802.11e.

IEEE 802.15.4 uses CSMA/CA mechanism that does not provide reliable solutions in the in-body sensor networks. The path loss inside human body results in improper Clear Channel Assessment (CCA). For a thresh-

Table 2. Packet delivery ratio and power (in $\mathrm{mW}$ ).

\begin{tabular}{|c|c|c|c|c|}
\hline \multicolumn{2}{|c|}{ Sensor Nodes } & \multirow{2}{*}{$\begin{array}{c}\text { IEEE } \\
802.15 .4 \\
100 \%\end{array}$} & \multirow{2}{*}{$\begin{array}{c}\text { IEEE } \\
802.11 \mathrm{e} \\
\left(\mathrm{AC} \_\mathrm{BE}\right)\end{array}$} & \multirow{2}{*}{$\begin{array}{c}\begin{array}{c}\text { IEEE } \\
802.11 \mathrm{e} \\
\left(\mathrm{AC}_{-} \mathrm{VO}\right)\end{array} \\
100 \%\end{array}$} \\
\hline Packet & Wave-form & & & \\
\hline $\begin{array}{l}\text { Delivery } \\
\text { Ratio }\end{array}$ & Parameter & $99.77 \%$ & $100 \%$ & $100 \%$ \\
\hline \multirow{2}{*}{$\begin{array}{c}\text { Power } \\
(\mathrm{mW})\end{array}$} & Wave-form & 1.82 & 4.01 & 3.57 \\
\hline & Parameter & 0.26 & 2.88 & 2.77 \\
\hline
\end{tabular}




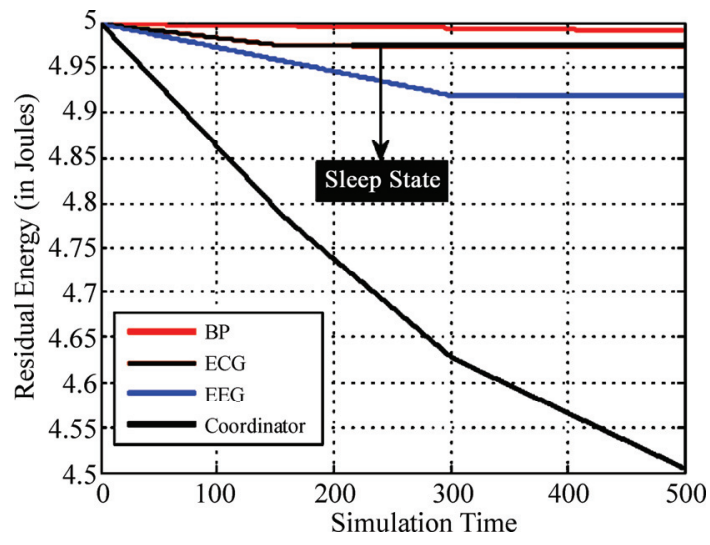

Figure 6. Residual emergency at on-body nodes.

old of $-85 \mathrm{dBm}$ and $-95 \mathrm{dBm}$, the on-body nodes cannot see the activity of in-body nodes when they are away at 3 meters distance from the body surface [37]. An alternative solution is to use TDMA-based protocols for a WBAN. Therefore, we analyze the performance of a preamble-based TDMA [38] protocol for an on-body sensor network. We use ns-2 [39] for extensive simulations. Figure 6 shows the residual energy at the on-body nodes and the coordinator. After the nodes finish their transmissions, they go into sleep mode. The ECG node sleeps after 150 seconds. When the EEG node finishes its transmission at 300 seconds, the coordinator consumes less energy as indicated by the slight change in the curve.

\section{WBAN Applications}

WBANs have great potential for several applications including remote medical diagnosis, interactive gaming, and military applications.
Table 3 shows some of the in-body and on-body applications [40]. In-body applications include, monitoring and program changes for pacemakers and implantable cardiac defibrillators, control of bladder function, and restoration of limb movement [41]. On-body medical applications include monitoring ECG, blood pressure, temperature, and respiration. Furthermore, on-body nonmedical applications include monitoring forgotten things, establishing a social network, and assessing soldier fatigue and battle readiness.

The following part discusses some of the WBAN applications:

1) Cardiovascular Diseases: Traditionally, holter monitors were used to collect cardio rhythm disturbances for offline processing without real-time feedback. However, transient abnormalities are sometimes hard to capture. For instance, many cardiac diseases are associated with episodic rather than continuous abnormalities, such as transient surges in blood pressure, paroxysmal arrhythmias or induced episodes of myocardial ischemia and their time cannot be accurately predicated [42]. A WBAN is a key technology to prevent the occurrence of myocardial infarction, monitor episodic events or any other abnormal condition and can be used for ambulatory health monitoring.

2) Cancer Detection: Cancer remains one of the biggest threats to the human life. According to National Center for Health Statistics, about 9 million people had cancer diagnosis in 1999 [43]. A set of miniaturised sensors capable of monitoring cancer cells can be seamlessly integrated in a WBAN. This allows physician to diagnose tumors without biopsy.

3) Asthma: A WBAN can help millions of patients suffering from asthma by monitoring allergic agents in the

Table 3. In-body and on-body sensor networks applications.

\begin{tabular}{|c|c|c|c|c|c|c|}
\hline $\begin{array}{l}\text { Application } \\
\text { Type }\end{array}$ & Sensor Node & Date Rate & $\begin{array}{c}\text { Duty Cycle } \\
\text { (per device)\% } \\
\text { per time }\end{array}$ & $\begin{array}{c}\text { Power } \\
\text { Consumption }\end{array}$ & $\begin{array}{c}\text { QoS } \\
\text { (Sensitive to Latency) }\end{array}$ & Privacy \\
\hline \multirow{3}{*}{$\begin{array}{c}\text { In-body } \\
\text { Applications }\end{array}$} & Glucose Sensor & Few Kbps & $<1 \%$ & Extremely Low & Yes & High \\
\hline & Pacemaker & Few Kbps & $<1 \%$ & Low & Yes & High \\
\hline & Endoscope Capsule & $>2 \mathrm{Mbps}$ & $<50 \%$ & Low & Yes & Medium \\
\hline \multirow{3}{*}{$\begin{array}{c}\text { On-body } \\
\text { Medical } \\
\text { Applications }\end{array}$} & ECG & $3 \mathrm{kbps}$ & $<10 \%$ & Low & Yes & High \\
\hline & $\mathrm{SpO} 2$ & $32 \mathrm{bps}$ & $<1 \%$ & Low & Yes & High \\
\hline & Blood Pressure & $<10$ bps & $<1 \%$ & High & Yes & High \\
\hline \multirow{3}{*}{$\begin{array}{c}\text { On-body } \\
\text { Non-Medical } \\
\text { Applications }\end{array}$} & Music for Headsets & $1.4 \mathrm{Mbps}$ & High & Relatively High & Yes & Low \\
\hline & $\begin{array}{l}\text { Forgotten Things } \\
\text { Monitor }\end{array}$ & $256 \mathrm{kbps}$ & Medium & Low & No & Low \\
\hline & Social Networking & $<200 \mathrm{kbps}$ & $<1 \%$ & Low & Low & High \\
\hline
\end{tabular}




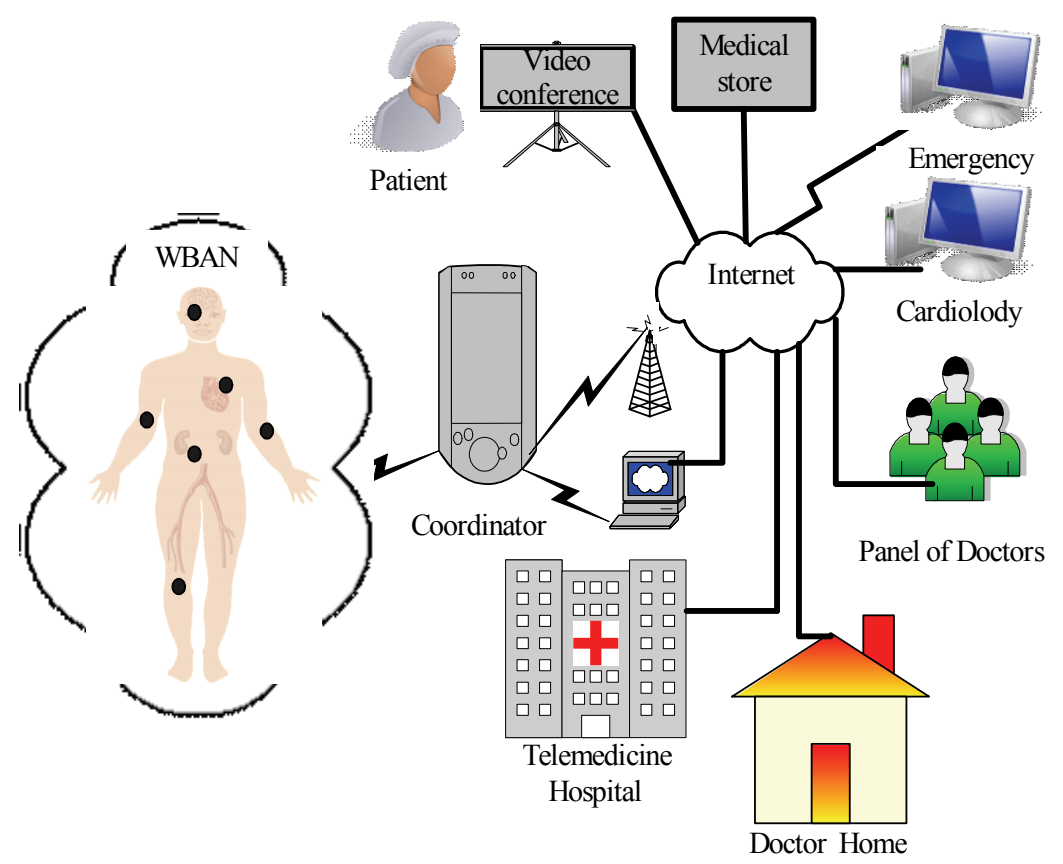

Figure 7. A real-time telemedicine infrastructure for patient rehabilitation.

air and providing real-time feedback to the physician. Chu et al proposed a GPS-based device that monitors environmental factors and triggers an alarm in case of detecting information allergic to the patient [44].

4) Telemedicine Systems: Existing telemedicine systems either use dedicated wireless channels to transfer information to the remote stations, or power demanding protocols such Bluetooth that are open to interference by other devices working in the same frequency band. These characteristics limit prolonged health monitoring. A WBAN can be integrated into a telemedicine system that supports unobtrusive ambulatory health monitoring for long period of time. Figure 7 shows a real-time telemedicine infrastructure for patient rehabilitation.

5) Artificial Retina: Retina prosthesis chips can be implanted in the human eye that assists patient with limited or no vision to see at an adequate level.

6) Battlefield: WBANs can be used to connect soldiers in a battlefield and report their activities to the commander, i.e., running, firing, and digging. The soldiers should have a secure communication channel in order to prevent ambushes.

\section{Conclusions}

In this paper, we proposed a WBAN infrastructure that supports on-demand, emergency, and normal traffic using wakeup and main radios. This infrastructure proves to be adequate for unobtrusive health monitoring. We further provided a technical discussion on the in-body antenna design and supported patch antenna for in-body communication. We also discussed low-power MAC protocol for a WBAN. Existing low-power MAC protocols have several limitations to accommodate the heterogeneous traffic in a reliable manner and hence require new power-efficient solutions. We finally outlined the potential of a WBAN for ubiquitous healthcare, entertainment, and military applications.

\section{References}

[1] J. G. Cleland, K. Swedberg, and F. Follath, "A survey of the quality of care among patients with heart failure in Europe. Part 1: Patient characteristics and diagnosis," The Euro Heart Failure Survey Programme, Euro Heart Journal, pg 24, pp. 442-463, 2003.

[2] http://www.foxnews.com/story/0,2933,142436,00.html Date Visited, 15 December 2008.

[3] Heart Failure Facts and Figures: OU Medical Centre.

[4] http://www.who.int/whosis/mort/profiles/mortwprokor repofkorea.pdf.

[5] A. Barroso, J. Benson, et al., "The DSYS25 sensor platform," In Proceedings of the ACM sensys, 2004.

[6] C. Borger, et al:: "Health spending projections through 2015: Changes on the horizon," In Health Affairs Web Exclusive W61, February 22, 2006.

[7] Electromagnetic compatibility and Radio spectrum Matters (ERM), ETSI TR 102 655, pg 21, 2008.

[8] http://fiji.eecs.harvard.edu/CodeBlue, Date Visited, 21 November 2008.

[9] http://www.mobihealth.org, Date Visited, 20 January 2009.

[10] http://www.cs.uoregon.edu/research/wearables/index.htm 
1, Date Visited, 21 July 2008.

[11] E. Jovanov, A. Milenkovic, C. Otto, and P. de Groen, "A wireless body area network of intelligent motion sensors for computer assisted physical rehabilitation," Journal of Neuro Engineering and Rehabilitation, Vol. 2, No. 6, March 2005.

[12] http://www.ubimon.net Date Visited, 16 March 2008.

[13] http://www.media.mit.edu/wearables/mithril, Date Visited, 09 February 2008.

[14] http://www.hitl.washington.edu, Date Visited, 25 April 2008.

[15] http://lifeguard.stanford.edu, Date Visited, 11 January 2009.

[16] http://www.ieee802.org/15/pub/TG6.html, Date Visited, 16 December 2008

[17] IEEE P1073.0.1.1/D01J, "Draft guide for health informatics-point-of-care medical device," Communicationtechnical report-Guidelines for the use of RF wireless technology, 2006.

[18] http://www.wireless-world-research.org/?id=92, Date Visited, 12 February 2009.

[19] G.-Z Yang, "Body sensor networks," Springer, pp. 117-143, 2006.

[20] J. Wojclk, et al., "Tissue recipe calibration requirements, SSI/DRB-TP-D01-003," Spectrum Sciences Institute RF Dosemetry Research Board, 51 Spectrum Way, Nepean, Ontario, K2R 1E6, Canada.

[21] H. M. Li, H Jindong H. Tan, "Heartbeat driven medium access control for body sensor networks," Proceedings of the 1st ACM SIGMOBILE, International Workshop on Systems and Networking Support for Healthcare and Assisted Living Environments, pp. 25-30, 2007.

[22] Braem, B. Latre, I. Moerman, C. Blondia, and P. Demeester, "The Wireless Autonomous Spanning tree Protocol for multihop wireless body area networks," in Proceedings of the First International Workshop on Personalized Networks. San Jose, California, USA, 2006.

[23] B. Latre, B. Braem, I. Moerman, C. Blondia, E. Reusens, W. Joseph, and P. Demeester, "A low-delay protocol for multihop wireless body area networks," Fourth Annual International Conference on Mobile and Ubiquitous Systems: Networking \& Services, Mobi Quitous, pp. 1-8, 6-10, 2007.

[24] H. M. Li and J. D. Tan, "Medium access control for body sensor networks," Computer Communications and Networks, ICCCN'07, pp. 210-215, 13-16 August 2007.

[25] N. F. Timmons and W. G. Scanlon, "Analysis of the performance of IEEE 802.15.4 for medical sensor body area networking," IEEE SECON, 2004.

[26] W. Ye, J. Heidemann, and D. Estrin, "Medium access control with coordinated adaptive sleeping for wireless sensor networks," IEEE Communications Letters, Vol. 12 No. 3, pp. 493-506, 2004.

[27] T. V. Dam and K. Langendoen, "An adaptive energyefficient MAC protocol for wireless sensor networks," in SenSys'03, Los Angeles, pp. 171-180, 2003.

[28] IEEE Std.802.15.4, "Wireless medium access control (MAC) and physical layer (PHY) specifications for low data rate wireless personal area networks (WPAN)," 2006.
[29] A. El-Hoiydi and J.-D. Decotignie, "WiseMAC: An ultra low power MAC protocol for the downlink of infrastructure wireless sensor networks," in the Proceedings of the Ninth IEEE Symposium on Computers and Communication, ISCC'04, Alexandria, Egypt, pp. 244-251, 2004.

[30] Technical Requirement Document, IEEE 802.15.6, January 2009.

[31] A. Sikora and V. Groza, "Coexistence of IEEE 802.15.4 with other systems in the $2.4 \mathrm{GHz}$ ISM-band," IEEE IMTC Proceeding, May 2005.

[32] N. Golmie, D. Cypher, and O. Rebala, "Performance analysis of low rate wireless technologies for medical applications," Computer Communications, Vol. 28, No. 10, pp. 1255-1275, June 2005.

[33] N. Chevrollier, N. Montavont, and N. Golmie, "Handovers and interference mitigation in healthcare environments," IEEE MILCOM Proceeding, October 2005.

[34] Howitt and J. Gutierrez, "IEEE 802.15.4 low rate-wireless personal area network coexistence issues," IEEE WNCN Proceeding, 2003.

[35] IEEE 802.11e Std, "Amendment to Part 11: Wireless LAN medium access control (MAC) and physical layer (PHY) specifications," Medium Access Control Quality of Services Enhancements, November 2005.

[36] D. Cavalcanti, R. Schmitt, and A. Soomro, "Performance analysis of 802.15.4 and 802.11e for body sensor network applications," 4th International Workshop on Wearable and Implantable Body Sensor Networks, BSN, 2007

[37] B. Zhen, H. B. Li, and R. Kohno, "IEEE body area networks and medical implant communications," Proceedings of the ICST 3rd International Conference on Body Area Networks, Tempe, Arizona, 2008.

[38] S. Ullah, R. Islam, et al., "Performance analysis of a preamble based TDMA protocol for wireless body area network", Journal of Communications Software and Systems, Vol. 4, No. 3, pp. 222-226, 2008.

[39] http://www.isi.edu/nsnam/ns, Date Visited, 17 March 2009.

[40] IEEE 15-08-0644-07-0006-TG6, Technical Requirements Document, 2008

[41] S. Ullah, H. Higgins, Y. W. Cho, H. S. Lee, and K. S. Kwak, "Towards RF communication and multiple access protocols in a body sensor network," JDCTA: International Journal of Digital Content Technology and its Applications, Vol. 2, No. 3, pp. 9-16, 2008.

[42] B. Lo and G. Z. Yang, "Key technical challenges and current implementations of body sensor networks," IEEE Proceedings of the 2nd International Workshop on Body Sensor Networks (BSN'05), pp. 1-5, April 2005.

[43] National Center for Health Statistics, URL: http://www. cdc.gov/nchs/Default.htm, Date Visited, 12 March 2009

[44] H.-T. Chu, C.-C. Huang, Z.-H. Lian, and T. J. P. Tsai, “A ubiquitous warning system for asthma-inducement," in IEEE International Conference on Sensor networks, Ubiquitous and Thrustworthy Computing, Taichung, Taiwan, pp. 186-191, 2006. 